\title{
Non-Relativistic Bound State Solutions of Modified Quadratic Yukawa plus q-Deformed Eckart Potential
}

\author{
Akaninyene D. Antia*, Ituen B. Okon, Akaninyene O. Akankpo, Jonah B. Usanga \\ Department of Physics, Faculty of Science, University of Uyo, Uyo, Nigeria \\ Email: ^antiacauchy@yahoo.com, *akaninyeneantia@uniuyo.edu.ng
}

How to cite this paper: Antia, A.D., Okon, I.B., Akankpo, A.O. and Usanga, J.B. (2020) Non-Relativistic Bound State Solutions of Modified Quadratic Yukawa plus $q$-Deformed Eckart Potential. Journal of Applied Mathematics and Physics, 8, 660-671.

https://doi.org/10.4236/jamp.2020.84051

Received: January 9, 2020

Accepted: March 31, 2020

Published: April 3, 2020

Copyright () 2020 by author(s) and Scientific Research Publishing Inc.

This work is licensed under the Creative

Commons Attribution International

License (CC BY 4.0).

http://creativecommons.org/licenses/by/4.0/

\begin{abstract}
We have obtained approximate bound state solutions of Schrödinger wave equation with modified quadratic Yukawa plus q-deformed Eckart potential Using Parametric Nikiforov-Uvarov (NU) method. However, we obtained numerical energy eigenvalues and un-normalized wave function using confluent hypergeometric function (Jacobi polynomial). With some modifications, our potential reduces to a well-known potential such as Poschl-Teller and exponential inversely quadratic potential. Numerical bound state energies were carried out using a well-designed Matlab algorithm while the plots were obtained using origin software. The result obtained is in agreement with that of the existing literature.
\end{abstract}

\section{Keywords}

Schrodinger Equation, Nikiforov-Uvarov Method, Modified Quadratic Yukawa Plus $q$-Deformed Eckart Potential

\section{Introduction}

Researchers have put on their interest over the years with the aim of investigating the bound state solutions of relativistic and nonrelativistic wave equations for different potentials. A few of these potentials have been solved exactly [1], while others can only be solved approximately [2] [3], with the use of different approximation schemes [4] [5]. Subsequently, various methods have been applied to obtain the solutions of the nonrelativistic wave equations with a chosen potential model. Methods include the factorization method [6], functional analysis approach [7] [8] [9], supersymmetry quantum mechanics (SUSYQM) [10], Nikiforov-Uvarov method (NU) [11] [12] and others. 
The Eckart potential is one of the most important exponential-type potentials in the literature and it has been widely used in physics and chemical physics [13] [14] [15]. Recently, [16] has employed the asymptotic iteration method to calculate any $\ell$-state solutions of the Schrödinger equation with the Eckart potential by proper approximation of the centrifugal term. Energy eigenvalues and corresponding eigen functions are obtained explicitly. The energy eigenvalues are calculated numerically for some values of $\ell$ and $n$.

The Yukawa potential, which is also known as the screened Coulomb potential, is greatly important with applications cutting across nuclear physics and condensed matter physics [17] [18]. The screened Coulomb potential is used mostly in short-range interactions [19] [20]. The Yukawa potential is known to be the potential of a charged particle in a weakly non-ideal plasma. It also describes the charged particle effects in a sea of conduction electrons in solid-state physics.

With approximate analytical solution of the Yukawa potential with arbitrary momenta using the Nikiforov-Uvarov method, they obtained approximate analytical solutions of the radial Schrödinger equation for the Yukawa potential, and the energy eigenvalues and the corresponding eigen functions are calculated in closed forms [21]. Some numerical results are presented and show that these results are in good agreement with those obtained previously by other methods. As the screening parameter tends to zero, the energy levels of Yukawa potential are equivalent to that of the familiar pure Coulomb potential. Eckart potential is one of the most important exponential type potentials for the study of nuclear forces [22] [23]. Basically, many properties of nuclear forces are explained using Yukawa potential and to predict the existence of pi-meson. This is the main reason why the study of the combination of the two potential models is very significant and highly essential. However, after the above mentioned studies on these different potentials and their lofty importance, we seek to investigate the bound state solutions of the Schrodinger equation with the modified quadratic Yukawa plus $q$-deformed Eckart potential of the form:

$$
V(r)=\frac{v_{o} \mathrm{e}^{-2 \alpha r}}{r^{2}}+v_{1}\left(\frac{1+\mathrm{e}^{-2 \alpha r}}{1-q \mathrm{e}^{-2 \alpha r}}\right)+v_{2}\left(\frac{4 \mathrm{e}^{-2 \alpha r}}{\left(1-q \mathrm{e}^{-2 \alpha r}\right)^{2}}\right),
$$

where $v_{o}, v_{1}, v_{2}$ are the potential depth, $q$ is the deformation parameter, $r$ is the inter-nuclear distance and $\alpha$ is the screening parameter. It can be deduced that when $v_{o}=v_{1}=0, v_{2}=-v_{1}$ and $q=-1$, the above combined potential reduces to the Poschl-Teller potential. When $v_{1}=v_{2}=0$, the potential reduces to Exponential inversely quadratic potential. Using the parametric NU method, we derive the energy bound state solutions and their wave functions of the Schrodinger equation for the modified quadratic Yukawa plus q-deformed Eckart potential, analytically and numerically. The rest of the manuscript is organized as follows: In Section 2, a review of Nikiforov-Uvarov method is presented. The radial solutions of Schrodinger equation are presented in Section 3. The numer- 
ical solutions are presented in Section 4 while discussion and conclusion are presented in Sections 5 and 6 respectively.

\section{Nikiforov-Uvarov Method: Parametric Method}

The parametric form is simply using parameters to obtain explicitly energy eigenvalues and it is based on the solutions of a generalized second order linear differential equation with special orthogonal functions [2]. The hypergeometric NU method has shown high utility in calculating the exact energy levels of all bound states for some solvable quantum systems.

Given a second order differential equation of the form

$$
\psi^{\prime \prime}(s)+\frac{\bar{\tau}}{\sigma(s)} \psi^{\prime}(s)+\frac{\bar{\sigma}(s)}{\sigma^{2}(s)} \psi(s)=0
$$

where $\sigma$ and $\bar{\sigma}$ are polynomials at most degree and $\bar{\tau}$ is first degree polynomials. The parametric generalization of the $\mathrm{N}-\mathrm{U}$ method is given by the generalized hypergeometric-type equation

$$
\psi^{\prime \prime}(s)+\frac{c_{1}-c_{2} s}{s\left(1-c_{3} s\right)} \psi^{\prime}+\frac{1}{s^{2}\left(1-c_{3} s\right)^{2}}\left[-\chi_{1} s^{2}+\chi_{2} s-\chi_{3}\right] \psi(s)=0
$$

Thus Equation (2) can be solved by comparing it with Equation (3) and the following polynomials are obtained

$$
\bar{\tau}(s)=\left(c_{1}-c_{2} s\right), \sigma(s)=s\left(1-c_{3} s\right), \bar{\sigma}(s)=-\chi_{1} s^{2}+\chi_{2} s-\chi_{3}
$$

The parameters obtainable from Equation (3) serve as an important tool for finding the energy eigenvalue and eigenfunctions.

Where $c_{1}, c_{2}$ and $c_{3}$ are parametric constants, other parametric constants are given below

$$
\left\{\begin{array}{l}
c_{4}=\frac{1}{2}\left(1-c_{1}\right) \\
c_{5}=\frac{1}{2}\left(c_{2}-2 c_{3}\right) \\
c_{6}=c_{5}^{2}+\chi_{1} \\
c_{7}=2 c_{4} c_{5}-\chi_{2} \\
c_{8}=c_{4}^{2}+\chi_{3} \\
c_{9}=c_{3} c_{7}+c_{3}^{2} c_{8}+c_{6} \\
c_{10}=c_{1}+2 c_{4}+2 \sqrt{c_{8}} \\
c_{11}=c_{2}-2 c_{5}+2\left[\sqrt{c_{9}}+c_{3} \sqrt{c_{8}}\right] \\
c_{12}=c_{4}+\sqrt{c_{8}} \\
c_{13}=c_{5}-\left[\sqrt{c_{9}}+c_{3} \sqrt{c_{8}}\right]
\end{array}\right.
$$

The energy equation is given as

$$
c_{2} n-(2 n+1) c_{5}+(2 n+1)\left[\sqrt{c_{9}}+c_{3} \sqrt{c_{8}}\right]+n(n+1) c_{3}+c_{7}+2 c_{3} c_{8}+2 \sqrt{c_{8} c_{9}}=0 \text { (5) }
$$

The total wave function is given by 


$$
\psi(s)=\phi(s) \chi_{n}(s)=N_{n} s^{c_{12}}\left(1-c_{3} s\right)^{c_{13}} P^{\left[c_{10}, c_{11}\right]}\left(1-2 c_{3} s\right)
$$

\section{The Radial Solutions of Schrödinger Equation of the Proposed Potential}

The Schrödinger equation is given by

$$
\frac{\mathrm{d}^{2} R}{\mathrm{~d} r^{2}}+\left[\frac{2 \mu}{\hbar^{2}}\left[E_{n l}-V(r)\right]-\frac{l(l+1)}{r^{2}}\right] R(r)=0
$$

where $\mu$ is the reduced mass, $E_{n l}$ is the rotational vibrational energy spectra of the diatomic molecules, $\hbar$ is the reduced Planck's constant, $n$ and $l$ are the radial and orbital angular momentum quantum numbers, respectively [7] [14].

Substituting Equation (1) into (7) gives

$\frac{\mathrm{d}^{2} R}{\mathrm{~d} r^{2}}+\left[\frac{2 \mu}{\hbar^{2}}\left[E-\frac{v_{o} \mathrm{e}^{-2 \alpha r}}{r^{2}}-v_{1}\left(\frac{1+\mathrm{e}^{-2 \alpha r}}{1-q \mathrm{e}^{-2 \alpha r}}\right)-v_{2}\left(\frac{4 \mathrm{e}^{-2 \alpha r}}{\left(1-q \mathrm{e}^{-2 \alpha r}\right)^{2}}\right)\right]-\frac{l(l+1)}{r^{2}}\right] R(r)=0$

Let's define suitable approximation to the centrifugal term as

$$
\frac{1}{r^{2}}=\frac{4 \alpha^{2} \mathrm{e}^{-2 \alpha r}}{\left(1-q \mathrm{e}^{-2 \alpha r}\right)^{2}}
$$

Substituting Equation (9) into (8) gives

$$
\begin{aligned}
& \frac{\mathrm{d}^{2} R}{\mathrm{~d} r^{2}}+\left\{\frac{2 \mu}{\hbar^{2}}\left[E-\frac{4 \alpha^{2} v_{o} \mathrm{e}^{-4 \alpha r}}{\left(1-q \mathrm{e}^{-2 \alpha r}\right)^{2}}-v_{1}\left(\frac{1+\mathrm{e}^{-2 \alpha r}}{1-q \mathrm{e}^{-2 \alpha r}}\right)-v_{2}\left(\frac{4 \mathrm{e}^{-2 \alpha r}}{\left(1-q \mathrm{e}^{-2 \alpha r}\right)^{2}}\right)\right]\right. \\
& \left.-\frac{4 \alpha^{2} \mathrm{e}^{-2 \alpha r}}{\left(1-q \mathrm{e}^{-2 \alpha r}\right)^{2}} l(l+1)\right\} R(r)=0
\end{aligned}
$$

In order to present Equation (10) in a form that is conformable to Equation (3) then, there is a need for the transformation

$$
\mathrm{s}=q \mathrm{e}^{-2 \alpha r}=\frac{\mathrm{d}^{2} R}{\mathrm{~d} r^{2}}=4 \alpha^{2} q^{2} \mathrm{e}^{-4 \alpha r} \frac{\mathrm{d}^{2} R}{\mathrm{ds}^{2}}+4 \alpha^{2} q \mathrm{e}^{-2 \alpha r} \frac{\mathrm{d} R}{\mathrm{ds}} \text { and } \mathrm{e}^{-2 \alpha r}=\frac{s}{q}
$$

Substituting (11) into (10) and simplifying gives

$$
\begin{aligned}
& \frac{\mathrm{d}^{2} R}{\mathrm{~d} s^{2}}+\frac{q(1-s)}{q s(1-s)} \frac{\mathrm{d} R}{\mathrm{~d} s}+\frac{1}{s^{2} q^{2}(1-s)^{2}}\left[-\left(\varepsilon^{2} q^{2}-A^{2}+B^{2} q\right) s^{2}\right. \\
& \left.+\left(2 \varepsilon^{2} q^{2}+B^{2} q^{2}-B^{2} q-C^{2} q-q \lambda\right) s-\left(\varepsilon^{2} q^{2}+B^{2} q^{2}\right)\right] R(s)=0
\end{aligned}
$$

where

$$
\frac{\mu E}{2 \alpha^{2} \hbar^{2}}=-\varepsilon^{2}, \frac{2 \mu v_{o}}{\hbar^{2}}=A^{2}, \frac{\mu v_{1}}{2 \alpha^{2} \hbar^{2}}=B^{2}, \frac{\mu v_{2}}{\alpha^{2} \hbar^{2}}=C^{2} \text { and } l(l+1)=\lambda
$$

Comparing with parametric $\mathrm{NU}$

$$
\left\{\begin{array}{l}
\chi_{1}=\varepsilon^{2} q^{2}-A^{2}+B^{2} q, \chi_{2}=2 \varepsilon^{2} q^{2}+B^{2} q^{2}-B^{2} q-C^{2} q-q \lambda \\
\chi_{3}=\varepsilon^{2} q^{2}+B^{2} q^{2}, c_{1}=c_{2}=c_{3}=1
\end{array}\right.
$$




$$
\left\{\begin{array}{l}
c_{4}=\frac{1}{2}\left(1-c_{1}\right)=0 \\
c_{5}=\frac{1}{2}\left(c_{2}-2 c_{3}\right)=-\frac{1}{2} \\
c_{6}=c_{5}^{2}+\chi_{1}=\frac{1}{4}+\varepsilon^{2} q^{2}-A^{2}+B^{2} q \\
c_{7}=2 c_{4} c_{5}-\chi_{2}=-2 \varepsilon^{2} q^{2}-B^{2} q^{2}+B^{2} q+C^{2} q+q \lambda \\
c_{8}=c_{4}^{2}+\chi_{3}=\varepsilon^{2} q^{2}+B^{2} q^{2} \\
c_{9}=c_{3} c_{7}+c_{3}^{2} c_{8}+c_{6}=2 B^{2} q+C^{2} q+q \lambda+\frac{1}{4}-A^{2} \\
c_{10}=c_{1}+2 c_{4}+2 \sqrt{c_{8}}=1+2 \sqrt{\varepsilon^{2} q^{2}+B^{2} q^{2}}=1+2 \varepsilon q+2 \sqrt{B^{2} q^{2}} \\
c_{11}=c_{2}-2 c_{5}+2\left[\sqrt{c_{9}}+c_{3} \sqrt{c_{8}}\right]=2+2\left[\sqrt{2 B^{2} q+C^{2} q+q \lambda+\frac{1}{4}-A^{2}}+\sqrt{\varepsilon^{2} q^{2}+B^{2} q^{2}}\right. \\
c_{12}=c_{4}+\sqrt{c_{8}}=\sqrt{\varepsilon^{2} q^{2}+B^{2} q^{2}} \\
c_{13}=c_{5}-\left[\sqrt{c_{9}}+c_{3} \sqrt{c_{8}}\right]=-\frac{1}{2}-\sqrt{2 B^{2} q+C^{2} q+q \lambda+\frac{1}{4}-A^{2}}-\sqrt{\varepsilon^{2} q^{2}+B^{2} q^{2}}
\end{array}\right]
$$

\subsection{Energy of the Proposed Potential}

The energy can be calculated by substituting Equation (14) and Equation (15) into (5) with simple mathematical algebraic simplification. Hence, the energy eigen equation become

$$
\begin{aligned}
& n-(2 n+1)\left(-\frac{1}{2}\right)+(2 n+1)\left[\sqrt{2 B^{2} q+C^{2} q+q \lambda+\frac{1}{4}-A^{2}}+\sqrt{\varepsilon^{2} q^{2}+B^{2} q^{2}}\right] \\
& +n^{2}-n+\left(-2 \varepsilon^{2} q^{2}-B^{2} q^{2}+B^{2} q+C^{2} q+q \lambda\right)+2 \varepsilon^{2} q^{2} \\
& +2 B^{2} q^{2}+2 \sqrt{\left(\varepsilon^{2} q^{2}+B^{2} q^{2}\right)\left(2 B^{2} q+C^{2} q+q \lambda+\frac{1}{4}-A^{2}\right)}=0
\end{aligned}
$$

Factorizing and rearranging Equation (16)

$$
\begin{aligned}
& -\left[n^{2}+n+\frac{1}{2}+(2 n+1) \sqrt{2 B^{2} q+C^{2} q+q \lambda+\frac{1}{4}-A^{2}}+(2 n+1) \sqrt{B^{2} q^{2}}\right. \\
& \varepsilon=\frac{\left.+B^{2} q+C^{2} q+q \lambda+B^{2} q^{2}+\sqrt{B^{2} q^{2}\left(2 B^{2} q+C^{2} q+q \lambda+\frac{1}{4}-A^{2}\right)}\right]}{q(2 n+1)+2 \sqrt{q^{2}\left(2 B^{2} q+C^{2} q+q \lambda+\frac{1}{4}-A^{2}\right)}} \\
& E_{n l}=-\frac{2 \alpha^{2} \hbar^{2}}{\mu}\left[\begin{array}{c}
n^{2}+n+\frac{1}{2}+(2 n+1) \sqrt{2\left(\frac{\mu v_{1}}{2 \alpha^{2} h^{2}}\right) q+\left(\frac{\mu v_{2}}{\alpha^{2} h^{2}}\right) q+q l(l+1)+\frac{1}{4}-\left(\frac{2 \mu v_{o}}{h^{2}}\right)} \\
+(2 n+1) \sqrt{\left(\frac{\mu v_{1}}{2 \alpha^{2} h^{2}}\right) q^{2}+\left(\frac{\mu v_{1}}{2 \alpha^{2} \hbar^{2}}\right) q+\left(\frac{\mu v_{2}}{\alpha^{2} \hbar^{2}}\right) q+q l(l+1)+\left(\frac{\mu v_{1}}{2 \alpha^{2} \hbar^{2}}\right) q^{2}} \\
+\sqrt{\left(\frac{\mu v_{1}}{2 \alpha^{2} \hbar^{2}}\right) q^{2}\left(2\left(\frac{\mu v_{1}}{2 \alpha^{2} \hbar^{2}}\right) q+\left(\frac{\mu v_{2}}{\alpha^{2} \hbar^{2}}\right) q+q l(l+1)+\frac{1}{4}-\left(\frac{2 \mu v_{o}}{\hbar^{2}}\right)\right)} \\
q(2 n+1)+2 \sqrt{q^{2}\left(2\left(\frac{\mu v_{1}}{2 \alpha^{2} \hbar^{2}}\right) q+\left(\frac{\mu v_{2}}{\alpha^{2} \hbar^{2}}\right) q+q l(l+1)+\frac{1}{4}-\left(\frac{2 \mu v_{o}}{\hbar^{2}}\right)\right)}
\end{array}\right]
\end{aligned}
$$




\subsection{Deductions from the Energy Equation}

Case 1:

If we replace parameter $v_{o}=v_{1}=0, v_{2}=-v_{1}$ and $q=-1$, then the potential function reduces to Poschl-Teller potential which is written as:

$$
V(r)=\frac{-4 v_{1} \mathrm{e}^{-2 \alpha r}}{\left(1+\mathrm{e}^{-2 \alpha r}\right)^{2}}
$$

The energy equation for Poschl-Teller potential becomes:

$$
E_{n l}=-\frac{2 \alpha^{2} \hbar^{2}}{\mu}\left[\frac{n^{2}+n+\frac{1}{2}+(2 n+1) \sqrt{\left(\frac{\mu v_{1}}{\alpha^{2} \hbar^{2}}\right)-l(l+1)+\frac{1}{4}+\left(\frac{\mu v_{1}}{\alpha^{2} \hbar^{2}}\right)-l(l+1)}}{(2 n+1)+2 \sqrt{\left(\frac{\mu v_{1}}{\alpha^{2} \hbar^{2}}\right)-l(l+1)+\frac{1}{4}}}\right]^{2}
$$

\section{Case 2:}

If we also replace parameter $v_{1}=v_{2}=0$ we obtain exponential inversely quadratic potential which is expressed as

$$
V(r)=\frac{v_{0} \mathrm{e}^{-2 \alpha r}}{r^{2}}
$$

Also, the energy equation becomes:

$$
E_{n l}=-\frac{2 \alpha^{2} \hbar^{2}}{\mu}\left[\frac{n^{2}+n+\frac{1}{2}+(2 n+1) \sqrt{q l(l+1)+\frac{1}{4}-\left(\frac{2 \mu v_{o}}{h^{2}}\right)}+q l(l+1)}{q(2 n+1)+2 \sqrt{q^{2}\left(+q l(l+1)+\frac{1}{4}-\left(\frac{2 \mu v_{o}}{\hbar^{2}}\right)\right)}}\right]^{2}
$$

\subsection{The Wave Function of the Proposed Potential}

The wave function for the proposed potential can be calculated using equation (6), expressed in terms of Jacobi polynomial and substituting the necessary parameters. Thus,

$$
\begin{aligned}
& \psi(s)=\Phi(s) x_{n}(s) \\
& =N_{n} s \sqrt{-\frac{\mu E}{2 \alpha^{2} \hbar^{2}} q^{2}+\frac{\mu v_{1}}{2 \alpha^{2} \hbar^{2}} q^{2}}(1-s)\left[-\frac{1}{2}-\sqrt{\frac{\mu v_{1}}{2 \alpha^{2} \hbar^{2}} q+\frac{\mu v_{2}}{\alpha^{2} \hbar^{2}} q+q l(l+1)+\frac{1}{4}-\frac{2 \mu v_{o}}{\hbar^{2}}}-\sqrt{-\frac{\mu E}{2 \alpha^{2} \hbar^{2}} q^{2}+\frac{\mu v_{1}}{2 \alpha^{2} \hbar^{2}} q^{2}}\right] \\
& \times P^{\left[1+2 \sqrt{-\frac{\mu E}{2 \alpha^{2} \hbar^{2}}} q+2 \sqrt{\frac{\mu v_{1}}{2 \alpha^{2} \hbar^{2}} q^{2}}, 2+2\left[\sqrt{2 \frac{\mu v_{1}}{2 \alpha^{2} \hbar^{2}} q+\frac{\mu v_{2}}{\alpha^{2} \hbar^{2}} q+q l(l+1)+\frac{1}{4}-\frac{2 \mu v_{o}}{\hbar^{2}}}+\sqrt{-\frac{\mu E}{2 \alpha^{2} \hbar^{2}} q^{2}+\frac{\mu v_{1}}{2 \alpha^{2} \hbar^{2}} q^{2}}\right]\right]}(1-2 s)
\end{aligned}
$$

where $N_{n}$ is the normalization constant.

\section{The Numerical Computation for Energy Equation of the Proposed Potential Using Schrödinger Equation}

The numerical computation was carried out using MATLAB 8.0 software while graphical plot was carried out using origin software. The computation parameters have been incorporated in the numerical table. The energy equation for the proposed potential is given in Equation (18). The computation was carried for various range of values of the screening i.e. for large and small values of $\alpha$. 


\section{Discussion}

The results of the numerical computation that were carried out for the screening parameter $\alpha=(0.1$ to 0.5$)$ with the potential depths $V_{0}=0.3, V_{1}=0.1, V_{2}=0.2$. Tables 1-5 show that for $\alpha=(0.1$ to 0.5$)$, the numerical bound state energy decreases with an increase in quantum state. It is also observed that the energy increases with a decrease in screening parameter $\alpha$. However, the energy spectral diagram as shown in Figures 1-5 shows unique quantization of different energy levels with respect to the quantum state. The numerical bound state energies obtained for the various values of $\alpha$ are predominantly negative which the sufficient and necessary condition for bound state solutions. Also the negative bound state eigenvalues obtained show that the potential is suitable for describing particle state and their bound state properties.

\section{Conclusion}

We used the Nikiforov-Uvarov method to compute bound state solution of non-relativistic wave equation. We developed a new potential model which is the

Table 1. Numerical computation for $v_{0}=0.3, \hbar=1.0, \mu=1.0, v_{1}=0.1, v_{2}=0.2, q=1.0$.

\begin{tabular}{ccccc}
\hline$n$ & $E_{n l}(\alpha=0.1), l=0$ & $E_{n l}(\alpha=0.1), l=1$ & $E_{n l}(\alpha=0.1), l=2$ & $E_{n l}(\alpha=0.1), l=3$ \\
\hline 0 & -0.358723860422 & -0.373451796993 & -0.402514181567 & -0.445267610477 \\
1 & -0.475369012878 & -0.491714416155 & -0.523889432048 & -0.571048112966 \\
2 & -0.600647379426 & -0.618682417516 & -0.654093357336 & -0.705803142808 \\
3 & -0.734864206154 & -0.754635641966 & -0.793364191204 & -0.849724805080 \\
4 & -0.878257368475 & -0.899795273869 & -0.941895045046 & -1.002973867370 \\
5 & -1.031005151520 & -1.054328978440 & -1.099835318390 & -1.165677990550 \\
6 & -1.193240706270 & -1.218363132520 & -1.267299510740 & -1.337937137990 \\
7 & -1.365064290600 & -1.391993618710 & -1.444375701660 & -1.519829600630 \\
8 & -1.546552317100 & -1.575293981950 & -1.631132241580 & -1.711417064680 \\
\hline
\end{tabular}

Table 2. Numerical computation for $v_{0}=0.3, \hbar=1.0, \mu=1.0, v_{1}=0.1, v_{2}=0.2, q=1.0$.

\begin{tabular}{ccccc}
\hline$n$ & $E_{n l}(\alpha=0.2), l=0$ & $E_{n l}(\alpha=0.2), l=1$ & $E_{n l}(\alpha=0.2), l=2$ & $E_{n l}(\alpha=0.2), l=3$ \\
\hline 0 & -0.433780559429 & -0.494363866152 & -0.610559494829 & -0.776706656632 \\
1 & -0.681533081008 & -0.755505128052 & -0.894725310230 & -1.089765618020 \\
2 & -0.965541546776 & -1.053407891020 & -1.216334999480 & -1.440874970160 \\
3 & -1.287362985910 & -1.389313601350 & -1.576247051710 & -1.830596392810 \\
4 & -1.647812113560 & -1.763917890310 & -1.974991302070 & -2.259310793850 \\
5 & -2.047347843110 & -2.177631633140 & -2.412903173260 & -2.727276909810 \\
6 & -2.486246379460 & -2.630710863480 & -2.890202843360 & -3.234674069180 \\
7 & -2.964683306910 & -3.123322720630 & -3.407039979430 & -3.781629551740 \\
8 & -3.482775365680 & -3.655580683110 & -3.963519540590 & -4.368235808860 \\
\hline
\end{tabular}


Table 3. Numerical computation for $v_{0}=0.3, \hbar=1.0, \mu=1.0, v_{1}=0.1, v_{2}=0.2, q=1.0$.

\begin{tabular}{ccccc}
\hline$n$ & $E_{n l}(\alpha=0.3), l=0$ & $E_{n l}(\alpha=0.3), l=1$ & $E_{n l}(\alpha=0.3), l=2$ & $E_{n l}(\alpha=0.3), l=3$ \\
\hline 0 & -0.523052525651 & -0.661370123967 & -0.919655127988 & -1.28258956659 \\
1 & -0.913253413467 & -1.097274964830 & -1.423624712520 & -1.86403452990 \\
2 & -1.388776992580 & -1.619048677850 & -2.014441440220 & -2.53317685538 \\
3 & -1.952153981160 & -2.228660567050 & -2.693363583100 & -3.29078021582 \\
4 & -2.604372373890 & -2.927007369790 & -3.461069410510 & -4.13731637556 \\
5 & -3.345895349130 & -3.714551928370 & -4.317951065080 & -5.07308614428 \\
6 & -4.176968473750 & -4.591556423970 & -5.264249578690 & -6.09828854546 \\
7 & -5.097733958340 & -5.558180233760 & -6.300120794220 & -7.21305986496 \\
8 & -6.108279821150 & -6.614525733030 & -7.425669769950 & -8.41749630564 \\
\hline
\end{tabular}

Table 4. Numerical computation for $v_{0}=0.3, \hbar=1.0, \mu=1.0, v_{1}=0.1, v_{2}=0.2, q=1.0$.

\begin{tabular}{ccccc}
\hline$n$ & $E_{n l}(\alpha=0.4), l=0$ & $E_{n l}(\alpha=0.4), l=1$ & $E_{n l}(\alpha=0.4), l=2$ & $E_{n l}(\alpha=0.4), l=3$ \\
\hline 0 & -0.627591541101 & -0.874606577279 & -1.32739264725 & -1.95796715215 \\
1 & -1.167338531860 & -1.522463516870 & -2.11456444317 & -2.89392389718 \\
2 & -1.865350921070 & -2.326614313680 & -3.05852731571 & -3.98749113984 \\
3 & -2.722717879280 & -3.289031117730 & -4.16067450669 & -5.23951982221 \\
4 & -3.739774576790 & -4.410499607300 & -5.42170064184 & -6.65050810572 \\
5 & -4.916658364750 & -5.691393534650 & -6.84198775364 & -8.22076276659 \\
6 & -6.253435948480 & -7.131913188680 & -8.42176256885 & -9.95048181780 \\
7 & -7.750143631940 & -8.732175506250 & -10.1611679045 & -11.8397983528 \\
8 & -9.406802865400 & -10.49225332580 & -12.0602981088 & -13.8888049619 \\
\hline
\end{tabular}

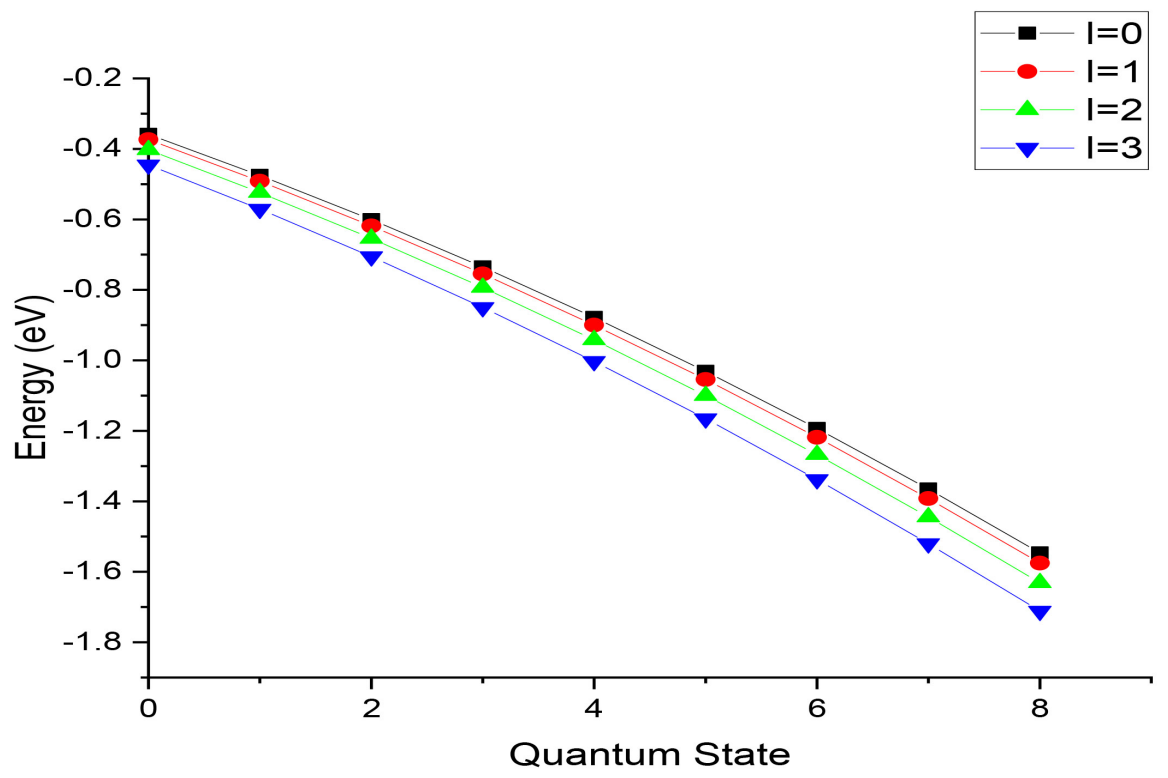

Figure 1. Energy spectral diagram with Schrödinger equation for $\alpha=0.1$. 
Table 5. Numerical computation for $v_{0}=0.3, \hbar=1.0, \mu=1.0, v_{1}=0.1, v_{2}=0.2, q=1.0$.

\begin{tabular}{ccccc}
\hline$n$ & $E_{n l}(\alpha=0.5), l=0$ & $E_{n l}(\alpha=0.5), l=1$ & $E_{n l}(\alpha=0.5), l=2$ & $E_{n l}(\alpha=0.5), l=3$ \\
\hline 0 & -0.749317899709 & -1.13386318743 & -1.83234253525 & -2.80079527677 \\
1 & -1.439799855380 & -2.03415440794 & -2.96841426538 & -4.17870059279 \\
2 & -2.388456731380 & -3.18228093061 & -4.35154186289 & -5.80426205934 \\
3 & -3.589627738370 & -4.57947155574 & -5.98306986743 & -7.67835319798 \\
4 & -5.041897269490 & -6.22617321021 & -7.86363513976 & -9.80146692921 \\
5 & -6.744744343520 & -8.12258737350 & -9.99357686299 & -12.1738997806 \\
6 & -8.697932398990 & -10.2688182127 & -12.3730919053 & -14.7958400397 \\
7 & -10.90133853290 & -12.6649249983 & -15.0023022605 & -17.6674127310 \\
8 & -13.35489256650 & -15.3109439381 & -17.8812875111 & -20.7887040137 \\
\hline
\end{tabular}



Figure 2. Energy spectral diagram with Schrödinger equation for $\alpha=0.2$.

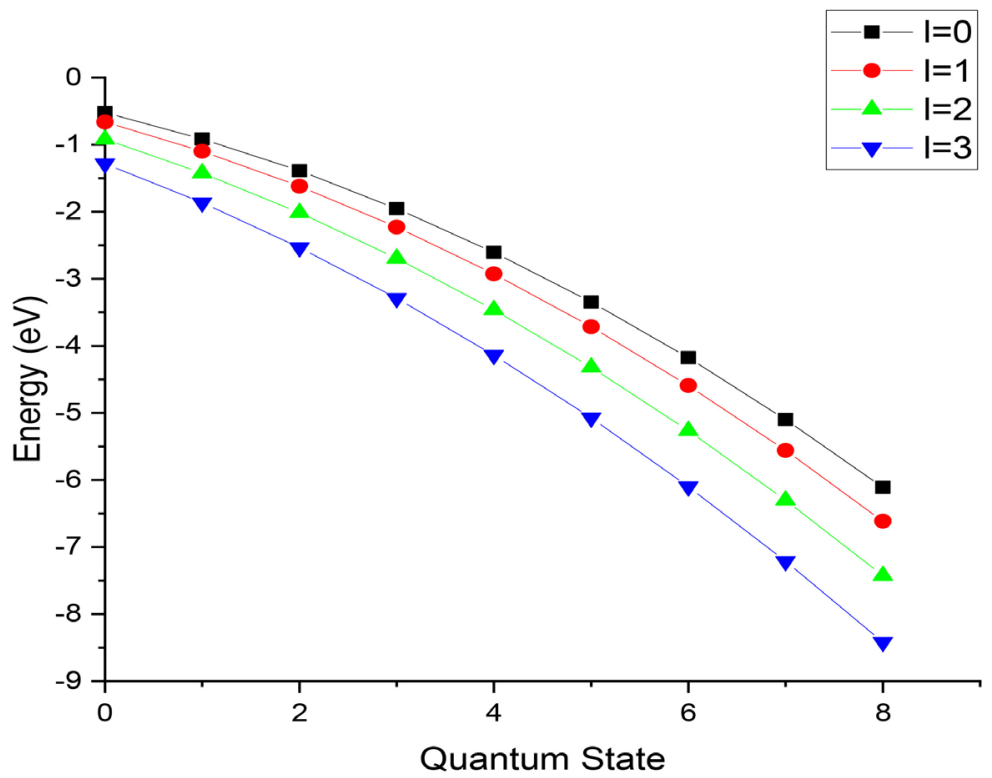

Figure 3. Energy spectral diagram with Schrödinger equation for $\alpha=0.3$. 


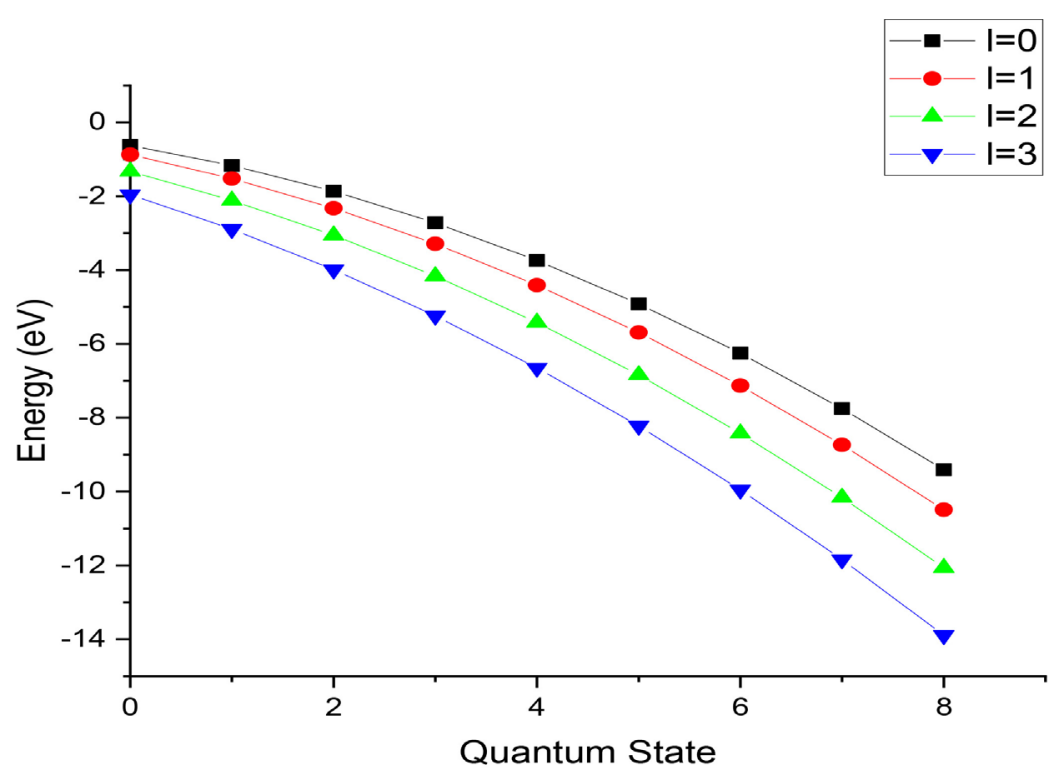

Figure 4. Energy spectral diagram with Schrödinger equation for $\alpha=0.4$.

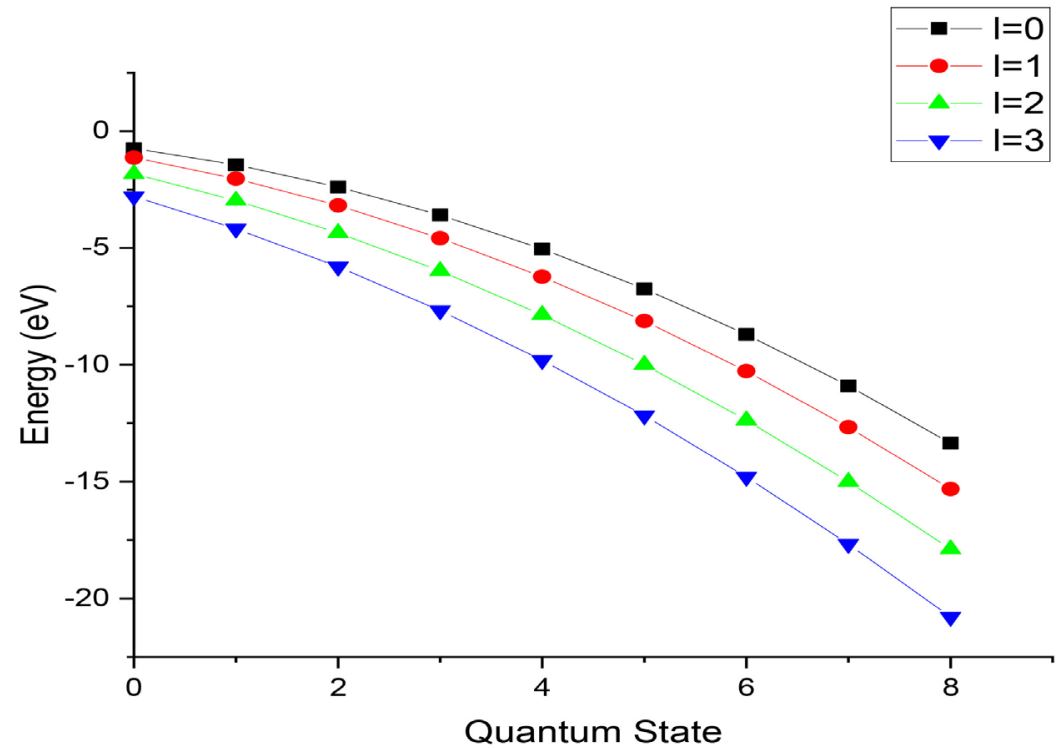

Figure 5. Energy spectral diagram with Schrödinger equation for $\alpha=0.5$.

modified quadratic Yukawa plus q-deformed Eckart potential. This potential reduces to a well-known potential called the Poschl-Teller potential and the exponential inversely quadratic potential. We also implement some algorithms to carry out numerical computation for the resulting energy equations for various values of screening parameters. Our analytic and computational results for the proposed potential are in agreement with the existing literature.

\section{Acknowledgements}

The authors are very grateful to the reviewer for the significant suggestions which have led to the improvement of this article. 


\section{Conflicts of Interest}

The authors have no conflict of interest as regarding the publication of this article.

\section{References}

[1] Ikot, A., Akpabio, E. and Umoren, B. (2011) Exact Solutions of Schrodinger Equation with Inverted Woods-Saxon and Manning-Rosen Potential. Journal of Scientific Research, 3, 25-33. https://doi.org/10.3329/jsr.v3i1.5310

[2] Okon, I., Popoola, O. and Ituen, E. (2016) Bound State Solution to Schrödinger Equation with Hulthen plus Exponential Coulombic Potential with Centrifugal Potential Barrier Using Parametric Nikiforovuvarov Method. International Journal of Recent advances in Physics, 2, 5-7. https://doi.org/10.14810/ijrap.2016.5101

[3] Louis, H., Ita, B., Amos, P. and Phillip, P. (2018) Bound State Solutions of Klein-Gordon Equation with Manning-Rosen Plus a Class of Yukawa Potential Using Pekeris-Like Approximation of the Coulomb Term and Parametric Nikiforov-Uvarov. International Journal of Chemical Physical Science, 1, 7. https://doi.org/10.30731/ijcps.7.1.2018.33-37

[4] Berkdemir, C. and Han, J. (2005) Any l-State Solutions of Morse Potential through the Pekeris Approximation and Nikiforov-Uvarov Method. Chemical Physics Letters, 409, 203-207. https://doi.org/10.1016/j.cplett.2005.05.021

[5] Qiang, W. and Dong, S. (2007) Arbitrary l-State Solutions of the Rotating Morse Potential through the Exact Quantization Rule Method. Physics Letters A, 363, 169. https://doi.org/10.1016/j.physleta.2006.10.091

[6] Dong, S. (2017) Factorization Method in Quantum Mechanics. Springer, Amsterdam.

[7] Liu,Y., Zhang, G. and Jia, S. (2013) Calculation of the Interaction Potential Energy Curve and Vibrational Levels for the a3 2 ut State of Li27 Molecules. Physics Letters A, 377, 1444-1447.

[8] Tang, H., Liang, C., Zhang, H., Zhao, F. and Jia, C. (2014) Diatomic Molecule Energies of the Modified Rosen-Morse Potential Energy Model. Canadian Journal of Chemistry, 92, 341. https://doi.org/10.1139/cjc-2013-0563

[9] Jia, S. and Jia, Y. (2017) Relativistic Rotation-Vibrational Energies for the $\mathrm{CS}_{2} \mathrm{Mo-}$ lecule. The European Physical Journal D, 71, 3. https://doi.org/10.1140/epjd/e2016-70415-y

[10] Miftahul, M., Naufal, I., Suparmi, A. and Cari, C. (2019) Exact Solution of Schrödinger Equation in Cylindrical Coordinates for Double Ring-Shaped Coulomb Oscillator Potential Using SUSYQM Method. Journal of Physics. Conference Series, 1, 1127. https://doi.org/10.1088/1742-6596/1127/1/012005

[11] Antia, A., Christian, C. and Akpabio, L. (2018) Solutions of Schrödinger Equation with the Harmonic Oscillator Potential (HOP) in Cylindrical Basis. Physics and Astronomy International Journal, 2, 3. https://doi.org/10.15406/paij.2018.02.00084

[12] Parmar, H. (2019) Generalized Improved Non-Central Potential and Solution of Schrödinger Equation with Extended Ring-Shaped Potential through Nikiforov-Uvarov Method. The European Physical Journal Plus, 3, 134. https://doi.org/10.1140/epjp/i2019-12513-6

[13] Falaye, B. (2012) Any $\ell$-State Solutions of the Eckart Potential via Asymptotic Iteration Method. Central European Journal of Physics, 10, 960-965. https://doi.org/10.2478/s11534-012-0047-6 
[14] Edet, O., Okorie, S., Ngiangia, T. and Ikot, A. (2019) Bound State Solutions of the Schrodinger Equation for the Modified Kratzer Potential plus Screened Coulomb Potential. Indian Journal of Physics, 93, 1171-1179. https://doi.org/10.1007/s12648-019-01477-9

[15] Dong, S., Sun, H. and Dong, H. (2013) Arbitrary l-Wave Solution of the Schrodinger Equation for the Screen Coulomb Potential. International Journal of Modern Physics E, 22, Article ID: 1350036. https://doi.org/10.1142/S0218301313500365

[16] Ikhdair, S. and Sever, R. (2007) Polynomial Solutions of the Mie-Type Potential in the Dimensional Schrodinger Equation. Journal of Molecular Structure, 855, 13-17. https://doi.org/10.1016/j.theochem.2007.12.044

[17] Liverts, Z., Drukarev, G., Krivec, R. and Mandelzweig, B. (2008) Analytic Presentation of a Solution of the Schrodinger Equation. Few-Body Systems, 44, 367. https://doi.org/10.1007/s00601-008-0328-1

[18] Maghsoodi, E., Hassanabadi, H. and Aydodu, O. (2012) Dirac Particles in the Presence of the Yukawa Potential plus a Tensor Interaction in SUSYQM Frame Work. Physica Scripta, 86, Article ID: 015005. https://doi.org/10.1088/0031-8949/86/01/015005

[19] Ikhdair, M. and Sever, R. (2009) Exact Quantization Rule to the Kratzer-Type Potentials: An Application to the Diatomic Molecules. Journal of Materials Chemistry, 45, 1137. https://doi.org/10.1007/s10910-008-9438-8

[20] Taskin, F. and Kocak, G. (2010) Approximate Solutions of Schrodinger Equation for Eckart Potential with Centrifugal Term. Chinese Physics B, 9, Article ID: 090314. https://doi.org/10.1088/1674-1056/19/9/090314

[21] Hamzavi, M.,Thylwe, K. and Rajabi, A. (2012) Approximate Analytical Solution of the Yukawa Potential with Arbitrary Angular Momenta. Chinese Physics Letters, 29, Article ID: 080302. https://doi.org/10.1088/0256-307X/29/8/080302

[22] Eckart, C. (1930) The Penetration of Potential Banner by Electrons. Physical Review, 35, 1303. https://doi.org/10.1103/PhysRev.35.1303

[23] Weiss, J. (1964) Mechanism of Proton Transfer in Acid-Base Reaction. The Journal of Chemical Physics, 41, 1120. https://doi.org/10.1063/1.1726015 